\title{
Application of Graph Theory to the elaboration of personal genomic data for genealogical research
}

Vincenzo Palleschi, Luca Pagani, Stefano Pagnotta, Giuseppe Amato, Sergio Tofanelli

IIn this communication a representation of the links between DNA-relatives based on Graph Theory is applied to the analysis of personal genomic data to obtain genealogical information. The method is tested on both simulated and real data and its applicability to the field of genealogical research are discussed. We envisage the proposed approach as a valid tool for a streamlined application to the publicly available data generated by many online personal genomic companies. By this way, anonymized matrices of pairwise genome sharing counts will enable to improve the retrieval of genetic relationship between customers who provided explicit consent to the treatment of their data. 
1 Application of Graph Theory to the Elaboration of Personal Genomic

2

3

4

5

6

\title{
Data
}

\author{
for Genealogical Research
}

\author{
Vincenzo Palleschi ${ }^{1,2}$, Luca Pagani ${ }^{3,4+}{ }^{+}$, Stefano Pagnotta ${ }^{1}$, Giuseppe Amato ${ }^{5}$, Sergio Tofanelli ${ }^{6}$ \\ ${ }^{1}$ Institute of Chemistry of Organometallic Compounds \\ Research Area of National Research Council \\ Via G. Moruzzi, 1 - 56124 Pisa (ITALY) \\ ${ }^{2}$ Department of Civilizations and Forms of Knowledge \\ University of Pisa \\ Via G. Galvani, 1 - 56126 Pisa (ITALY) \\ ${ }^{3}$ Division of Biological Anthropology, University of Cambridge, UK \\ ${ }^{4}$ Department of Biological, Geological and Environmental Sciences, \\ University of Bologna \\ Via Selmi 3, 40126 Bologna (ITALY) \\ ${ }^{5}$ Institute of Sciences and Technology of Information \\ Research Area of National Research Council \\ Via G. Moruzzi, 1 - 56124 Pisa (ITALY) \\ ${ }^{6}$ Department of Biology \\ University of Pisa \\ Via L. Ghini, 13 - 56126 Pisa (ITALY)
}

\section{ABSTRACT}

In this communication a representation of the links between DNA-relatives based on Graph Theory is applied to the analysis of personal genomic data to obtain genealogical information. The method is tested on both simulated and real data and its applicability to the field of genealogical research are discussed. We envisage the proposed approach as a valid tool for a streamlined application to the publicly available data generated by many online personal genomic companies. By this way, anonymized matrices of pairwise genome sharing counts will enable to improve the retrieval of genetic relationship between customers who provided explicit consent to the treatment of their data.

Keywords: DNA Analysis, Personal Genomics, Genealogy, Genetic Genealogy, Statistical methods, Graph Theory, Ancestry reconstruction. 
$39+{ }^{\dagger}$ Corresponding Author: Luca Pagani - Ip.lucapagani@gmail.com 


\section{Introduction}

In recent years, a number of companies started offering commercial services based on DNA analysis for genealogical research ${ }^{[1-3]}$. The informatic tools available to interpret such results, usually provided by the same companies or by external services ${ }^{[4]}$, are mainly focused on general population studies (Paternal and Maternal lineages based on $Y$ chromosome and mitochondrial haplogroups, Ancestry Composition/Admixture, etc.). On the other hand, very few tools are provided to investigate the links of one's DNA profile with the relatives made recognizable through personal genomic data. Notably, these pre-compiled tools are often the only way to access the data provided by the DNA testing companies for a panel of hundreds or thousands of individuals. Therefore, the starting point of any downstream analysis based on this kind of data can only rely on the semi-processed input provided by the aforementioned tools. The introduction by the genetic service providers of a wrapped application tool would facilitate users' interpretations and unearth hidden genealogical information. Such tool should enable to implement the mass of data each single DNA test makes available in an easy-to-grasp graphical form. This would be particularly useful to detect the provenience of distant autosomic DNA-relatives from either the paternal or the maternal lineage. In fact this task is often made difficult by the links that might exist between the two parental genealogies due to the custom in closed communities to marry between relatives, especially in the past.

Here we describe and annotate an artificial intelligence tool that helps exploiting the information provided to customers by genealogical genetic services. The original approach of this work is the use of cross-information about the links between the living DNA-relatives of the test user (TU) for obtaining hints about the possible connections with other individuals, in the absence of a-priori genetic or genealogic evidence. 
64

65

66

67

68

69

\section{Data}

We performed quality checks via using the theoretical amount of genome sharing (Supplementary Table 1) to simulate a similarity matrix (Supplementary Table 2) based on two identical, hypothetical genealogies each made by 10 samples (individuals 1-10 and 11-20, Supplementary Figure 1). Pairs including individuals not linked in the genealogy were given a random amount of genomic sharing comprised between 0 and $2 \mathrm{Mbp}$.

We then used actual genomic data, consensually provided and anonymously treated, and derived from the results obtained by a test-user (TU) from the personal genomic service $23 a n d M e^{[2]}$. Such results typically consist of summary statistics on about one million single nucleotide polymorphisms (SNPs) ${ }^{[5]}$.

A total of 120 anonymized individuals (progressively numbered with an ID from 1 to 120) were considered in the analyses. All of them are 'DNA-relatives' of the TU according to the 23andMe criteria and accepted the invitation to share their DNA information (excluding data related to health conditions). The raw data is available in Supplementary Table 3. Since this is a secondary analysis of pre-existing data and the samples are treated in an anonymised version we did not apply for an ethical clearance.

We also retrieved an additional genome sharing matrix from an independent test user (TU2, Supplementary Table 4) who agreed to donate the matrix s/he obtained from 23andMe to be processed anonymously, in compliance with the ethical considerations provided in the paragraph above. This second matrix was used solely as a mean of independent confirmation of the validity of the approach presented here.

As reference parameter we considered the total amount of autosomal DNA in common between pairs of individuals, calculated as the total length of shared SNP haplotype blocks in mega base-pairs (Mbp) units. This amount, once converted into proportion of shared genome, provides a rough estimate of the number of generations separating any two individuals, under a simple model of "infinite number of ancestors" (Supplementary Table 1). Information either on 
90

the relevant chromosomes where the match occurs, or on the number of segments in common was not used. This choice is justified by the fact that only a minimum percentage of the individuals considered shows DNA matches on more than one chromosome. Furthermore, the information about the specific segment of the chromosome where such match occurs is not easily obtainable from the data made available to the users by 23 andMe.

Using the Genome-Wide Comparison option in the 23andMe 'Family Traits' feature, the input data were prepared in the form of a symmetric square matrix $C$, whose $C(i, j)$ elements correspond to the total length of shared SNP haplotype blocks between the individual $i$ and the individual $j$, expressed in Mbp units. Most elements of the matrix are equal to zero, corresponding to the fact that the majority of the individuals does not result genetically related. The sparsity of the matrix $C(i, j)$ is visually shown in Figure 1 , where the white points indicate a mutual match of any magnitude between two individuals, and the black correspond to no genetic relation at all.

\section{Classification}

The matrix (Supplementary Table 3) depicted in Figure 1 can be alternatively interpreted as a correlation matrix, a covariance matrix, a similarity matrix ${ }^{[6]}$ or it can be transformed in a distance matrix ${ }^{[7]}$. Accordingly, the way to elaborate and manipulate the associated information varies depending on the interpretation tasks. Given that the statistical analysis is aimed at simplifying data outputs, a loss of information with respect to the original data has to be expected. The effectiveness of the analysis thus depends on the amount of 'interesting' information unearthed out of the bulk of 'redundant' information. It follows that different methods can be more or less effective according to what is considered, from time to time, interesting or redundant.

To this extent, a number of potential confounders must be considered when dealing with the available genetic similarity matrix. First of all the genetic information on which the analysis is 
116 based is intrinsically fuzzy, because of the uncertainty in the data obtained by the service

117 provider (a few 'no-called' SNPs should be routinely expected). Additionally, the presence of

118 identical by state (IBS) other than identical by descent (IBD) ${ }^{[8]}$ SNPs could potentially bias the 119 genealogical interpretation, especially the one associated with distant relationships (Most

120 Recent Common Ancestors distant more than 6/7 generations). Finally, as opposed to 121 uniparental markers, the diploid autosomic data combine information inherited from the 122 paternal and maternal genealogy that should be kept separated when tracing one's ancestry.

123 Therefore, the analysis must be performed using statistical techniques robust enough to sustain 124 these unavoidable uncertainties.

\section{Graph theory approach}

The ideal framework for studying the complex network of links between the DNArelatives of a TU is the Graph Theory ${ }^{[9,10]}$. This approach, widely used in Mathematics, Engineering, and Computer Science, allows the analysis and graphical representation of the links between different entities in a network. In synthesis, the Graph Theory represents the elements in a network as vertices (or nodes) connected by edges. Edges are often associated with a value representing a weight. In our case, the weight of an edge connecting two vertexes is related to the genetic distance between them. A couple of vertexes $a$ and $b$ can be connected, in principle, by more than one edge. Graphs can be generally oriented, so that the edge from $a$ to $b$ is different from that linking $b$ to $a$. In this way, the distance between the vertexes $a$ and $b$ can be different from the distance between $b$ and $a$ (a typical example is driving a car between two points in a city, where the traffic regulations might impose different routes for the direct and return trip, see figure 2 ).

The relation between the vertexes is usually represented in matrix form (adjacency matrix $\left.^{[11]}\right)$ where the elements out of the diagonal are the weights of the corresponding edges.

140 If the adjacency matrix is symmetric (the distance between two nodes is the same in both 141 directions) the resulting graph is called unoriented. 
In our scenario, the correlation matrix $C(i, j)$ between the DNA-relatives of the TU is interpreted as a symmetric adjacency matrix. Therefore, we will use unoriented graphs, implemented using the Matlab ${ }^{\circledR}$ code provided in Supplementary Materials.

\section{Results}

The preliminary runs we performed on the simulated data (Supplementary Table 2, Supplementary Figures 2-7) showed that, once the effect of the randomly introduced noise was taken into account, the Graph approach yielded the expected inter-individual relationships. Particularly, the introduction of a 3Mbp cutoff (Supplementary Figure 4) to remove genetic links arising from the randomly added genetic similarities ( $<=2 \mathrm{Mbp}$ ) managed to re-create the simulated scenario (Supplementary Figure 1). More stringent thresholds (up to $48 \mathrm{Mbp}$, Supplementary Figures 5-7) further simplified the picture, leaving as viable connections only the individuals with the closest relationships. Remarkably, the two simulated genealogies (individuals 1-10 and individuals 11-20) were treated in the exact same manner by our approach, hence showing its robustness. This exercise served as a proof of principle to show that the introduction of a cutoff to remove genetic links below a certain threshold is beneficial to the removal of noise. According to the obtained results, a cutoff between 3 and $6 \mathrm{Mbp}$ (Supplementary Figures 4 and 5) is sufficient to remove noise while keeping in the pictures genetic links up to seven generations. It follows that in situations where the available genetic data is made up only by distantly related individuals (i.e. more than seven generations), a cutoff between 3 and $6 \mathrm{Mbp}$ is sufficient to remove the background noise, while keeping the true genealogical information embedded in the data.

We then applied the Graph Theory approach to the empirical genomic data. In the dataset analysed here, the TU adjacency matrix (Supplementary Table 3) is described by an unweighted Graph with 120 vertexes (individuals) and 196 edges (DNA links between them). The graphical representation of the Graph described by this matrix is shown in Supplementary Figure 8.

The main network connects 100 vertexes ( $83 \%$ of the total) by 190 edges ( $97 \%$ of the total) and 
169

170

171

172

173

174

175

176

177

178

179

180

181

182

183

184

185

186

187

188

189

190

191

192

193

194

195

persons. A strict interpretation of Supplementary Figure 8 would thus bring to the conclusion that all the individuals belonging to the main group should be considered as somehow related, directly or indirectly, to all the other members of the group. To reduce this connectivity and to assign the various individuals to the TU paternal and maternal ancestries, a further treatment of the input data is thus necessary.

\subsection{Pruning}

As shown for the simulated genealogies, the strength of the DNA cross-links between the individuals can be used to reduce (prune) the connections highlighted in Supplementary Figure 8. Since all the 120 individuals included in this study are, by design, related with the TU, no information can be derived from those that are connected only to the TU. They are represented, in graphical form, as isolated vertexes with no edges associated. Therefore, these individuals can be safely removed from the adjacency matrix without any loss of information. Moreover, as already discussed in Section 3, spurious connections could be introduced by fuzziness of the genetic data and the occurrence of IBS SNPS. These connections can be excluded via the application of an upper threshold on the genetic distances between the individuals. The threshold amount of shared genome for a link to be considered 'real' (i.e., corresponding to IBD SNPS) can be easily converted into expected number of generations, using Supplementary Table 1.

Following the results on the simulated data, and given the abundance of strong genetic links within the TU similarity matrix, we chose to apply a more stringent threshold to increase the readability of the resulting Graph. Figure 3 shows the Graph where only the edge weights greater or equal to $24 \mathrm{Mbp}$ (roughly a 8 generations distance between the vertexes/individuals, see Supplementary Table 1) are considered.

Figure 3 corresponds to the idea of unconnected graph that we associate with the separation of the different ancestral lines of the TU. Surprisingly enough, when the results of the Graph Theory are compared with the pre-existing genealogical information on some of the matching individuals, it turns out that the two large groups correspond to relatives of the TU related to 
196 the maternal grandfather (at the center of the figure) and maternal grandmother (at the left).

197 Another small group of three individuals, at the right in figure 3, shows up, containing an

198 individual associated to the maternal grandmother's lineage of the TU (n.22). The two

199 individuals that can be identified with reasonable certainty as belonging to the paternal

200 grandfather's (n. 118) and grandmother's (n. 96) lineage of the TU, remains unconnected.

201 These results are summarized in Table 1. The individuals underlined and marked in bold are the

202 ones for whom a genealogical evidence exists, and therefore can be assigned with certainty to a

203 given lineage. The ones underlined and marked in italic, on the other hand, cannot be assigned

204 with similar certainty, although there are strong independent clues suggesting that they would

205 actually belong to that lineage.

206 The adoption of a conservative threshold (24 Mbp / approx. 8 generations distance $/ 3^{\text {rd }}-4^{\text {th }}$

207 cousin range) to define a link between the individuals produced the classification reported in

208 Table 1, which is robust and reliable. However, only 17 individuals over a total of 120 (110 with

209 at least one DNA match besides the TU) are attributed to the corresponding ancestral lineage.

210 Reducing the level of the threshold to $12 \mathrm{Mbp}$ (approx. 9 generations distance) increases the number of individuals that can be associated to the different groups (Supplementary Figure 9).

212 Individual 22 is now correctly associated to the maternal grandmother's group, along with the other members of his/her subgroup. Most importantly the graph now shows an additional

214 group of three individuals $(21,46$ and 118) that can be associated to the TU paternal grandfather's lineage, on the basis of independent genealogical information existing for individual 118.

217 Further lowering the threshold to $6 \mathrm{Mbp}$, the threshold tested on the simulations (approx. 10

218 generations distance, i.e. a $4^{\text {th }}-5^{\text {th }}$ cousin range, which is usually considered the lower limit for

219 having a significant DNA match between two individuals) allows to recover important 220 information, graphically represented in Figure 4.

221 From the analysis of figure 4 it is evident that after lowering the threshold to $6 \mathrm{Mbp}, \mathrm{a}$ connection appears between the two main groups. The key elements which are linked to both 
223 the groups (corresponding to the maternal grandparents of the TU) are individual 83 (initially 224 classified in the maternal GM group) which connects with individual 80 in the maternal GF 225 group), individual 61 of the maternal GM group which connects with individual 42 in the 226 maternal GF group, and individual 86 of the maternal GF group which connects with individual 22713 of the maternal GM group.

228

230

232

233

Lowering the threshold also increased the number of individuals associated to the paternal grandfather of the TU, which at this level formed a group of five persons $(118,46,21,6$ and 64$)$ connected by the same sub-graph, and recovered a new group of five individuals $(96,112,65$, 100 and 68) that can be associated to the TU paternal grandmother's lineage on the basis of independent genealogical information existing for individual 96.

The main information that can be derived by the comparison of the Graphs obtained using different thresholds on the edge weight is a classification of the individuals according to the different ancestral lineages, with increasing 'levels of confidence'. In that respect, Supplementary Figure 8 would give a minimum level of information, providing classification at the confidence level of the minimum match in the $C(i, j)$ matrix, which in our case is $2 \mathrm{Mbp}$, subsequently refined at higher thresholds of genomic sharing in Figure 3, Supplementary Figure 9 and Figure 4.

The most important results of this paper are shown in Table II, where the classification of the DNA-relatives of the TU is reported according to his maternal and paternal ancestral lineages, with the corresponding confidence level, or 'strength', in brackets. The individuals connecting the groups corresponding to the two maternal grandparents are assigned to both the groups and marked in gray.

The Graph Theory method here proposed is capable of reliably classifying 62 individuals at strength $6(\mathrm{Mbp})$ over a total of 110 DNA-relatives of the TU (56\%). Six other unclassified groups with more than two members can also be determined. Some of them could be connected to the main groups if additional information from new DNA relatives of the TU will become available in the future. 
250 We further validate our approach on an additional genomic matrix (TU2), without

251 (Supplementary Figure 10) and with the $6 \mathrm{Mbp}$ threshold (Supplementary Figure 11). The Graph

252 obtained without the threshold (Supplementary Figure 10) notably includes link with the

253 "Mendel family", a real genealogy made freely available by 23andMe after assigning it a mock

254 family name. Given the lack of known relationship between TU2 and the Mendel family we take

255 the existing link as further support for the need of a 6Mbp threshold when interpreting the

256 genetic results. The application of such a threshold (Supplementary Figure 11) indeed yields a

257 cleaner Graph, with marked separations between the putative paternal and maternal TU2

258 family branches.

259

260

261

262

263

264

265

266

267

268

269

\section{Conclusion}

The statistical method presented in this work can be usefully exploited for extracting genealogical information from genetic/genomic data. The input data are usually 'fuzzy' and, therefore, the methods used for their analysis should be robust enough for providing useful information. The approach proposed, based on the Graph representation of the adjacency matrix built from the mutual matches between the DNA-relatives of the test user, after the setting of a $6 \mathrm{Mbp}$ threshold fulfils this requirement. The method, for which the code is provided at the bottom of this paper, could be easily implementable by the genetic service providers for an easy visualization of the DNA-links existing between the customer and the other users of the service, at different levels of confidence. 


\section{References}

271 [1] https://genographic.nationalgeographic.com/

272 [2] https://www.23andme.com/

273 [3] https://www.familytreedna.com/

274 [4] http://www.gedmatch.com/

275 [5] Nachman, M. W. (2001) Single nucleotide polymorphisms and recombination rate in 276 humans, Trends in genetics, 17 (9), 481-485.

277 [6] Muni S. Srivastava, Methods of Multivariate Statistics (2002) Wiley.

278 [7] Smouse, P. E. and Long, J. C. (1992) Matrix correlation analysis in anthropology and 279 genetics, American Journal of Physical Anthropology, 35, 187-213.

280 [8] Stevens, E.L., Heckenberg, G., Roberson, E.D.O., Baugher, J.D., Downey, T.J., Pevsner, J. 281 (2011) Inference of relationships in population data using identity-by-descent and identity-by282 state, PLoS Genetics, 7 (9), art. no. e1002287.

283 [9] Bondy, A., Murty, U.S.R., Graph Theory (2008) Springer-Verlag London.

284 [10] Pavlopoulos, G. A., Secrier, M., Moschopoulos, C. N., Soldatos, T. G., Kossida, S., Aerts, J., 285 Schneider, R., Bagos, P. G. (2011) Using graph theory to analyze biological networks, BioData 286 Mining, 4:10.

287 [11] Gehlenborg, N., Wong, B. (2012) Points of View: Networks, Nature Methods, 9, 115. 
1

Visual representation of the correlation between the individuals considered in this work

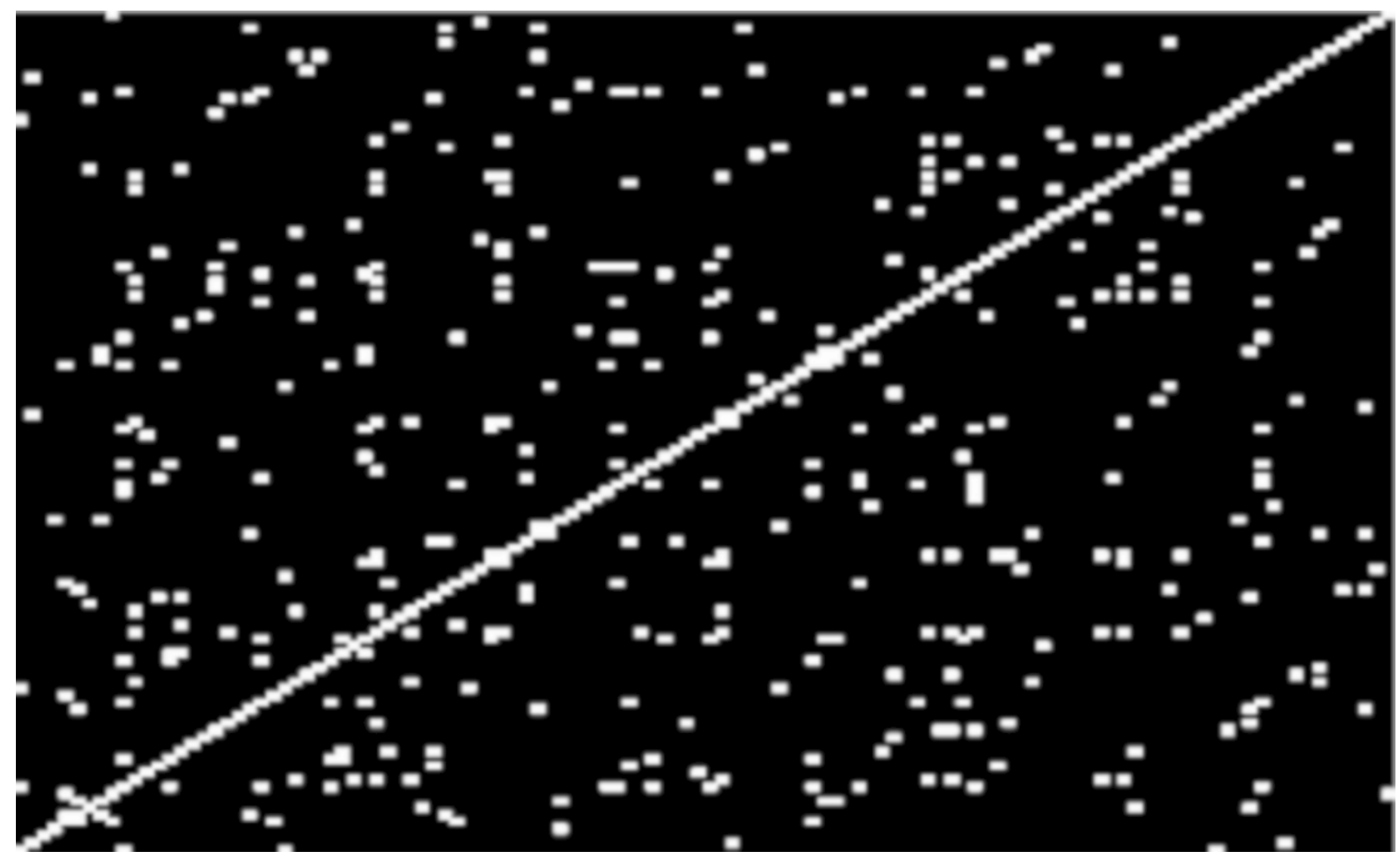


2

Graphic representation of a Graph with two vertexes and two edges (oriented Graph)

At the right, the corresponding adjacency matrix.
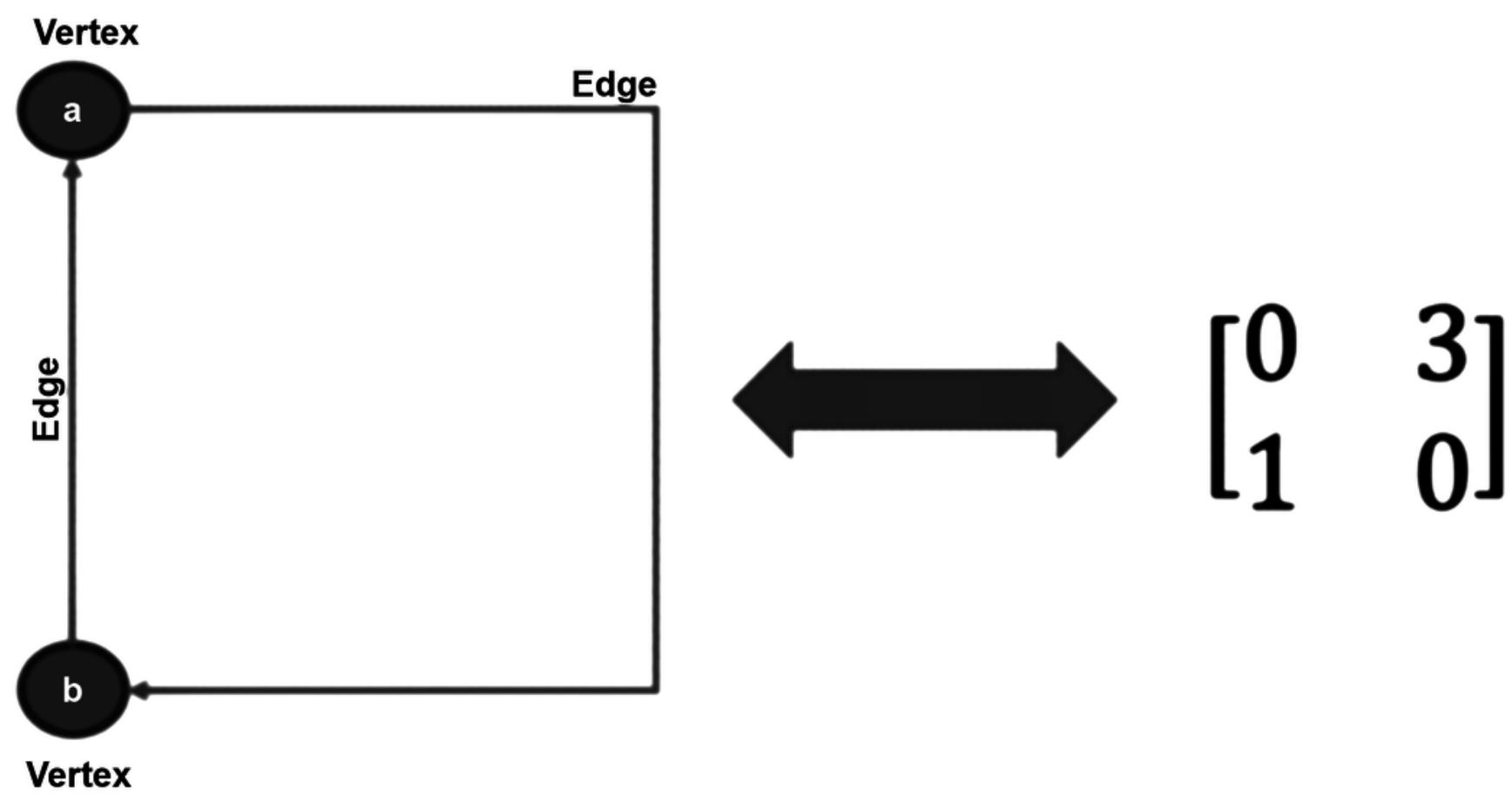
3

Graphic representation of the Graph described by the adjacency matrix $C(i, j)$ considering only the edges corresponding to DNA-matches greater or equal to $24 \mathrm{Mbp}$.

Isolated individuals and groups of two are not reported in the figure
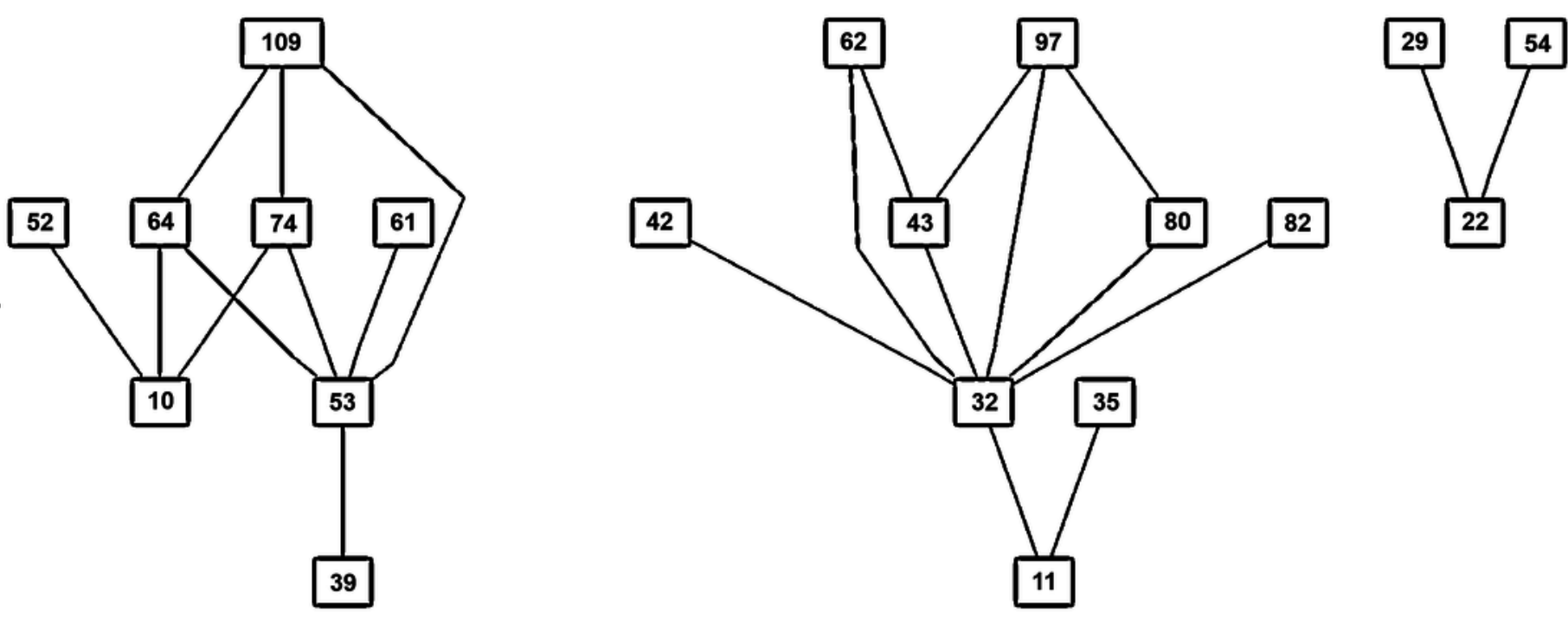


\section{4}

Graphic representation of the Graph described by the adjacency matrix $C(i, j)$ considering only the edges corresponding to DNA-matches greater or equal to $24 \mathrm{Mbp}$.

Isolated individuals and groups of two are not reported in the figure.

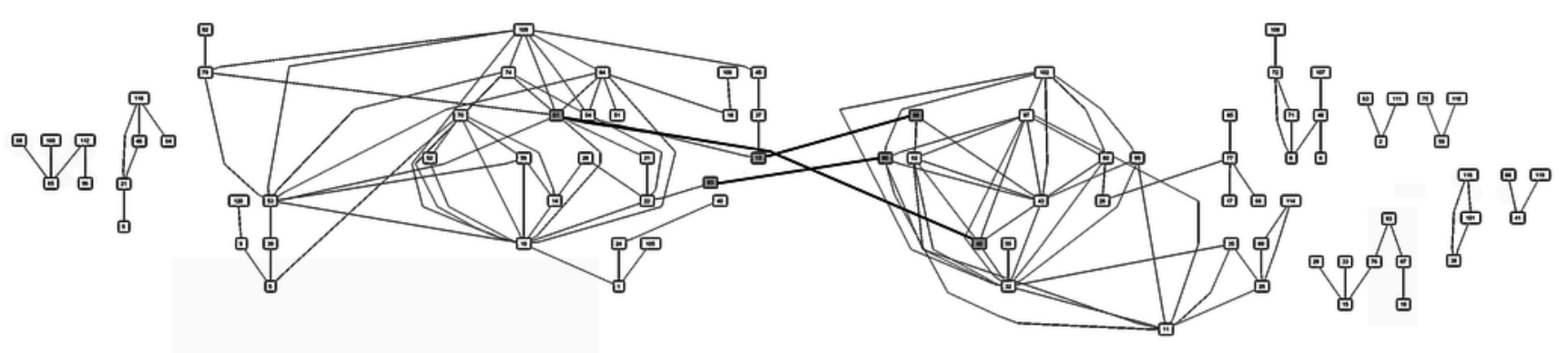




\section{Table $\mathbf{1}$ (on next page)}

Classification of the individuals according to their lineage (24 Mbp threshold).

Individuals underlined and marked in bold are the ones for whom a genealogical evidence exists, and therefore can be assigned with certainty to a given lineage. The ones underlined and marked in italic, on the other hand, cannot be assigned with similar certainty, although there are strong independent clues suggesting that they would actually belong to that lineage. 
1 Table 1 - Classification of the individuals according to their lineage ( $24 \mathrm{Mbp}$ threshold). Individuals 2 underlined and marked in bold are the ones for whom a genealogical evidence exists, and 3 therefore can be assigned with certainty to a given lineage. The ones underlined and marked in 4 italic, on the other hand, cannot be assigned with similar certainty, although there are strong 5 independent clues suggesting that they would actually belong to that lineage.

6

7

\begin{tabular}{|c|c|c|c|c|}
\hline Paternal GF & Paternal GM & Maternal GF & Maternal GM & Unclassified \\
\hline & & 62 & 52 & 28 \\
\hline & & 97 & 109 & 54 \\
\hline & & $\overline{42}$ & $\overline{84}$ & 22 \\
\hline & & $\overline{43}$ & 74 & \\
\hline & & 80 & $\overline{61}$ & \\
\hline & & 82 & 10 & \\
\hline & & 32 & 53 & \\
\hline & & 35 & 39 & \\
\hline & & $\overline{11}$ & & \\
\hline
\end{tabular}

10 


\section{Table 2 (on next page)}

Classification of the individuals according to their ancestral lineage

The corresponding level of confidence of the classification is reported in brackets. The individuals connecting the two groups of the maternal grandparents are marked in gray. 
1 Table 2 - Classification of the individuals according to their ancestral lineage. The corresponding level of 2 confidence of the classification is reported in brackets. The individuals connecting the two groups of the 3 maternal grandparents are marked in gray.

4

\begin{tabular}{|c|c|c|c|c|}
\hline Paternal GF & Paternal GM & Maternal GF & Maternal GM & Unclassified \\
\hline $118(12)$ & $\underline{96}(6)$ & $97(24)$ & $52(24)$ & 116 \\
\hline $46(12)$ & $112(6)$ & $62(24)$ & $109(24)$ & 101 \\
\hline $21(12)$ & $65(6)$ & $\underline{42}(24)$ & $84(24)$ & 38 \\
\hline $6(6)$ & $100(6)$ & $43(24)$ & $74(24)$ & ---------- \\
\hline \multirow[t]{30}{*}{$64(6)$} & $68(6)$ & $80(24)$ & $61(24)$ & 29 \\
\hline & & $82(24)$ & $10(24)$ & 33 \\
\hline & & $\underline{32}(24)$ & $53(24)$ & 15 \\
\hline & & $35(24)$ & $39(24)$ & 76 \\
\hline & & $11(24)$ & $70(12)$ & 93 \\
\hline & & $102(12)$ & $83(12)$ & 87 \\
\hline & & $95(12)$ & $54(12)$ & 19 \\
\hline & & $25(6)$ & $51(12)$ & ---------- \\
\hline & & $89(6)$ & $92(12)$ & 63 \\
\hline & & $114(6)$ & $79(12)$ & 111 \\
\hline & & $83(6)$ & $14(12)$ & 2 \\
\hline & & $61(6)$ & $\underline{22(12)}$ & ---------- \\
\hline & & $85(6)$ & $9(12)$ & 75 \\
\hline & & $77(6)$ & $5(12)$ & 110 \\
\hline & & $17(6)$ & $28(12)$ & 50 \\
\hline & & $66(6)$ & $1(6)$ & ---------- \\
\hline & & $26(6)$ & $24(6)$ & 88 \\
\hline & & $55(6)$ & $56(6)$ & 119 \\
\hline & & $13(6)$ & $42(6)$ & 41 \\
\hline & & $86(6)$ & $18(6)$ & ---------- \\
\hline & & & $106(6)$ & 108 \\
\hline & & & $31(6)$ & 72 \\
\hline & & & $13(6)$ & 71 \\
\hline & & & $86(6)$ & 8 \\
\hline & & & $45(6)$ & 48 \\
\hline & & & $37(6)$ & 4 \\
\hline & & & $120(6)$ & 107 \\
\hline & & & $105(6)$ & \\
\hline & & & $40(6)$ & \\
\hline & & & $80(6)$ & \\
\hline
\end{tabular}

